\title{
A DESCOBERTA DA TUBERCULOSE NO TERRITÓRIO: ANÁLISE QUALITATIVA DO TRABALHO DO AGENTE COMUNITÁRIO DE SAÚDE
}

\section{THE DISCOVERY OF TUBERCULOSIS IN TERRITORY: QUALITATIVE ANALYSIS OF WORK OF COMMUNITY HEALTH WORKERS}

\author{
EL DESCUBRIMIENTO DE LA TUBERCULOSIS EN EL TERRITORIO: \\ ANALISIS CUALITATIVO DEL TRABAJO DEL AGENTE COMUNITARIO \\ DE SALUD
}

\author{
Roxana Isabel Cardozo Gonzales* \\ JENIFER HARTER ${ }^{* *}$ \\ Ludmila Meireles Costa ${ }^{* * *}$ \\ Leandro Barbosa de Pinho ${ }^{* * * *}$ \\ LÍlian Moura De Lima ${ }^{* * * *}$ \\ Simone PieREN DOS ReIS ${ }^{* * * * *}$
}

\begin{abstract}
RESUMO
Objetivo: Analisar as ações de busca de casos de tuberculose no território pelo agente comunitário de saúde, bem como a sua articulação com o serviço de saúde para a continuidade das ações de diagnóstico da doença. Material e método: Estudo de abordagem qualitativa com doze agentes comunitários de saúde de quatro equipes de saúde da família de Pelotas que responderam a entrevista semiestruturada em julho de 2012. A análise do conteúdo das entrevistas seguiu os passos da Análise Temática. Resultados: O agente comunitário de saúde reconhece a necessidade do enfrentamento da situação social dos sintomáticos respiratórios e valoriza a formação de vínculo para a resolução das necessidades de saúde. Quanto à articulação com o serviço, identificaram-se fatores que limitam a sua efetivação, tais como a deficiência de estrutura e recursos humanos das unidades de saúde. Conclusão: Identificou-se que os agentes comunitários de saúde são atores fundamentais no controle da tuberculose, com potencial para construir pontes entre os serviços de saúde e a comunidade; além da capacidade de desenvolver uma abordagem baseada no vínculo, na responsabilização e resolutividade dos problemas
\end{abstract}

\footnotetext{
* Doutora em Saúde Publica pela EERP-USP. Docente Adjunto II do Departamento de Enfermagem da Faculdade de Enfermagem da Universidade Federal de Pelotas (UFPEL), Pelotas/RS, Brasil.Fax: 533221 1523.E-mail:rozanacardozoandre@ yahoo.com.br

${ }^{* *}$ Enfermeira. Mestre em Ciências pelo Programa de Pós-Graduação em Enfermagem (PPGEn/UFPEL). Doutoranda do PPGEn/UFPEL. Bolsista FAPERGS. Pelotas/RS. E-mail: jeniferharter@hotmail.com

${ }^{* * *}$ Enfermeira formada na Faculdade de Enfermagem da UFPEL. Pelotas/RS. Prefeitura Municipal do Rio Grande. E-mail: rozanacardozoandre@yahoo.com.br

${ }^{* * * *}$ Doutor em Enfermagem Psiquiátrica pela EERP-USP. Docente do Departamento de Assistência e Orientação Profissional da Escola de Enfermagem da Universidade Federal do Rio Grande do Sul (UFRGS), Porto Alegre/RS. E-mail: lbpinho@uol. com.br

${ }_{* * * * *}^{*}$ Enfermeira. Mestre pelo PPGEn/UFPEL. Doutoranda do PPGEn/UFPEL. Bolsista Capes/Demanda Social. Pelotas/RS. Endereço: Rua Santiago Dantas, 235 - casa 141, Três Vendas, Pelotas/RS. E-mail: lima.lilian@gmail.com

****** Enfermeira da Atenção Básica de Saúde de Pelotas. Mestranda do PPGEn/UFPEL. Pelotas/RS. E-mail: rozanacardozoandre@yahoo.com.br
} 
de saúde do indivíduo e família; e ainda produzir ações de saúde permeadas por aspectos culturais, sociais e de percepção do processo saúde doença, promovendo a união entre o conhecimento científico em saúde e as crenças locais.

Palavras chave: Tuberculose, atenção primária à saúde, agentes comunitários de saúde.

\begin{abstract}
Objective: To analyze the search actions of tuberculosis cases within the community health worker as well as its linkages with the health service to the continuity of the actions of diagnosis. Method: A qualitative study with twelve community health workers from four teams of family health in Pelotas who responded to the semi structured interview in July 2012. A content analysis of the interviews followed the steps of thematic analysis. Results: The community health worker recognizes the need for addressing the social situation of respiratory symptoms and values the bond for the resolution of health needs. Concerning the relationship with the service, identifying factors that limit its effectiveness, such as the disability in the structure and in the human resources of health facilities. Conclusion: It was found that the community health workers respondents are vital to the control of tuberculosis, with potential to build bridges between the health services and community, plus the ability to develop an approach based on relationship, accountability and solving health problems of individuals and family, and still produce health actions permeated by cultural, social and perceptions of health disease, promoting the union between the use of scientific knowledge in health and local beliefs.
\end{abstract}

Key words: Tuberculosis, primary health care, community health workers.

\title{
RESUMEN
}

Objetivo: Analizar las acciones de búsqueda de casos de tuberculosis en el territorio por el agente comunitario de salud y su articulación con el servicio de salud para la continuidad de acciones de diagnóstico de la enfermedad. Material y método: Estudio de abordaje cualitativo con doce agentes comunitarios de salud de cuatro equipos de salud de la familia de Pelotas/RS que respondieron entrevista semiestructurada en julio del 2012. El análisis de contenido de las entrevistas siguieron los pasos del Análisis Temático. Resultados: El agente comunitario de salud reconoce la necesidad de enfrentamiento de la situación social de los sintomas respiratorios y valoriza la formación de vínculo para la resolutividad de las necesidades de salud. Sobre la articulación con el servicio, se identificaron factores que la limitan, tales como deficiencia de estructura y recursos humanos de las unidades de salud. Conclusión: Se identificó que los agentes comunitarios de salud son actores fundamentales en el control de la tuberculosis, con potencial para construir puentes entre los servicios de salud y la comunidad; además de la capacidad de desarrollar un abordaje basado en el vínculo, en el compromiso y solución de problemas de salud del individuo y familia; y aún más, producir acciones de salud contemplando aspectos culturales, sociales y de percepción del proceso salud-enfermedad, promoviendo una unión entre el conocimiento científico en salud y las creencias locales.

Palabras clave: Tuberculosis, atención primaria de salud, agentes comunitarios de salud.

Fecha recepción: 20/05/13 Fecha aceptación: 03/08/15 


\section{INTRODUÇÃO}

A tuberculose (TB) tem se mantido como um problema de saúde pública no Brasil. Em 2013 a taxa nacional de incidência da doença foi de 35,4 casos por 100 mil habitantes (1), o que evidencia a necessidade da ênfase no desenvolvimento de ações para o controle da TB. Perante a importância epidemiológica da forma clínica pulmonar bacilífera, e do seu potencial de transmissibilidade, a detecção precoce destes casos é a estratégia primordial para o controle da doença. Para tanto, a busca dos sintomáticos respiratórios (SR) deve ser realizada de forma ativa e prioritariamente pelos serviços de atenção primária à saúde (APS), visto sua proximidade geográfica com a comunidade e pelo processo de trabalho caracterizado pelo desenvolvimento de ações no território $(2,3)$.

Neste cenário, os Agentes Comunitários de Saúde (ACS) são profissionais ímpares, que assumem protagonismo na execução das ações de identificação de SR na comunidade, no tratamento e na redução do abandono (4). Essa ação é altamente complexa, e requer conhecimento técnico específico, sobre sinais e sintomas da $\mathrm{TB}$, e a compreensão do contexto onde se produz a doença e se realiza a intervenção. Nesse sentido, o ACS, por ser membro da comunidade, pode proporcionar melhor inclusão e acolhimento do doente na rede de serviços, auxiliando-o na busca pelo diagnóstico, tornando-se o elo entre o serviço de saúde e o indivíduo (5).

A realização de visitas domiciliares (VD) situa os ACS no contexto sociocultural, possibilita a identificação das necessidades de saúde das famílias assistidas, e aproxima a equipe da Estratégia Saúde da Família (ESF) dos determinantes do processo saúde-doença (6).

Contudo, fatores de organização da rede de atenção básica de saúde nos municípios, como estruturas precárias dos serviços, e problemas quantitativos e qualitativos das equipes de saúde têm acarretado falhas que podem dificultar e até inviabilizar o controle da TB, interferindo, sobretudo, na busca de sintomáticos respiratórios (BSR) pelos ACS no território (5).

$\mathrm{Na}$ literatura atual, estudos predominantemente quantitativos evidenciam a relevância do ACS no contexto dos serviços de saúde, prioritariamente na realização de ações para detecção de casos de TB (5, 7-9). Considera-se, portanto, que há necessidade de maior aprofundamento com a realização de estudos qualitativos que suplementem o conhecimento existente sobre as diversas ações desse profissional no controle da TB.

Diante disso, levando-se em conta a situação epidemiológica da TB e a potencialidade do trabalho dos ACS na detecção dos SR, é fundamental analisar a atuação destes profissionais. Portanto, busca-se, nesse manuscrito, analisar as ações de busca de casos de TB no território pelo ACS, bem como sua articulação com o serviço de saúde para a continuidade das ações de diagnóstico da doença.

\section{MATERIAL E MÉTODO}

O conhecimento da realidade dos indivíduos da comunidade gera subsídios para os ACS planejarem intervenções mais efetivas no âmbito coletivo e individual. Visando conhecer mais amplamente o trabalho do ACS na BSR buscaram-se respostas numa abordagem qualitativa. Desse modo foi desenvolvido um estudo qualitativo, descritivo, do qual participaram 12 ACS de quatro Equipes de Saúde da Família (SF) de um município do interior do Estado do Rio Grande do Sul. A abordagem qualitativa, que se volta para os significados e a intencionalidade das ações nos contextos das estruturas sociais (10).

A amostra do estudo foi composta pela totalidade de ACS em atividade na unidade de saúde durante o período de coleta de dados. Utilizou-se como critério de exclusão 
aqueles que não estavam em atividade por afastamento de férias ou licença saúde durante a coleta de dados. Não houve negativas em participar do estudo.

Elegeu-se a técnica da entrevista semi-estruturada para a coleta de dados que consiste na combinação de um roteiro sistematizado com perguntas abertas e fechadas que permitem ao pesquisador se orientar ao elaborar as questões que pretende abordar (10).

Elaborou-se um roteiro de entrevista constituída de duas questões norteadoras: fale sobre as práticas de saúde do serviço em relação à busca de sintomáticos respiratórios de tuberculose da sua unidade de saúde e fale sobre os fatores de dificultam e facilitam as ações de busca de sintomáticos respiratórios em sua unidade de saúde.

Os dados foram gravados após previa concordância dos participantes do estudo, transcritos na íntegra, e agrupados por semelhança, formando uma unidade temática com dois núcleos de sentido (11).

Os profissionais foram identificados por codinomes para garantia do anonimato. No tratamento dos dados, foi utilizada a análise de conteúdo temática "descoberta do que está por trás dos conteúdos manifestos, indo além das aparências do que está sendo comunicado" (10). "Fazer uma análise temática, consiste em descobrir os 'núcleos de sentido' que compõem a comunicação e cuja presença, ou frequência de aparição pode significar alguma coisa para o objetivo analítico escolhido" (10).

Nesse procedimento analítico, "núcleo de sentido" é visto como uma unidade de significação no conjunto de uma comunicação. Neste estudo, os núcleos de sentido foram entendidos como ideias-eixo em torno das quais giram outras ideias. Assim, a pesquisa buscou trabalhar uma unidade temática (o ACS e a busca de sintomáticos respiratórios no território) e seus núcleos de sentido: A busca do sintomático respiratório: uma ação incorporada no cotidiano do ACS; Conexão entre o território e a rede de atenção à tu- berculose; $\mathrm{O}$ risco e a superação de medos e dificuldades.

A análise percorreu os seguintes passos: (a) leitura inicial procurando ter uma compreensão global do material; (b) identificação das unidades de significado que emergiram das falas dos entrevistados por unidade de saúde; (c) descoberta de núcleos de sentido e (e) interpretação e discussão dos núcleos de sentido encontrados.

O projeto foi aprovado pelo Comitê de Ética em Pesquisa da Faculdade de Enfermagem da Universidade Federal de Pelotas, sob parecer No116/2010, protocolo interno No 74/2010. Os profissionais entrevistados concordaram em participar através da assinatura do Termo de Consentimento Livre e Esclarecido.

\section{RESULTADOS}

$\mathrm{Na}$ busca de alcançar o objetivo proposto neste estudo, as falas foram agrupadas conforme os núcleos de sentido que apresentavam e deram origem às seguintes temáticas: A busca do sintomático respiratório: uma ação incorporada no cotidiano do ACS; Conexão entre o território e a rede de atenção à tuberculose; O risco e a superação de medos e dificuldades.

\section{A busca do sintomático respiratório: uma ação incorporada no cotidiano do ACS}

$\mathrm{O}$ relato mostra que a investigação do SR não acontece mediante a abordagem e questionamento direto da pessoa com sintomas relacionados à TB. Utiliza-se do diálogo com conteúdos voltados para identificação de problemas ou doenças na família. Esta abordagem inicial possibilita ao ACS investigar a presença dos sintomas da TB e o período de aparecimento.

Na visita domiciliar, tu vai, por exemplo, se tem 
um hipertenso [...], tem outras pessoas morando, tu não vai lá e pergunta só do hipertenso, tu pergunta da família toda [...] está com muita tosse [...] uma gripe geralmente nessa época mas aí se tu vê que aquela tosse continua, muito tempo, se começa com febre, suor noturno, aquela coisa que a gente sabe, tu vai atrás para enfermeira avaliar [...] (ACS No 12).

O ACS busca também orientar sobre os passos para o diagnóstico da doença valorizando-os no discurso. Contudo, em algumas situações, o ACS desconhece a presença de pessoas com sintomas nas famílias por ele acompanhadas.

A gente têm que ter uma certa perspicácia para perceber [...] a gente que encaminha, olha o bom é fazer os exames, para ver se é, mas tem toda aquela coisa, aquele preconceito, então geralmente tem que estar bem atenta, para observar, na verdade pelo tempo que a gente visita as famílias [...] conhece bem o pessoal, sabe se está acontecendo alguma coisa [...] têm alguns que a agente nem sabe, a gente fica sabendo através do serviço (ACS, No 5).

A abordagem à família no território requer o estabelecimento do vínculo entre o ACS e o doente e sua família. O vínculo permeia o trabalho do ACS e potencializa sua responsabilização com a saúde das famílias.

Trata-se de um profissional que busca e se articula com a UBS, providenciando insumos para coleta do escarro como uma forma de contribuir no diagnóstico da TB. Contudo, identificou-se nas falas que o estabelecimento do vínculo nem sempre resulta na aceitabilidade das orientações e encaminhamentos realizados.

O que facilita é o vínculo que tu tens com a família. [...] quando se tem um bom vínculo [...] se tem liberdade em falar com a pessoa, encaminhar se a pessoa esta com tosse há mais de uma semana e tem os sintomas ( $A C S N^{\circ} 5$ ).

[...] eu entro na casa, eu converso, eu já sei que eu posso estar até arriscando pegar ou levar para minha casa [a TB], mas aquele paciente se sente bem, ele conversa [...] (ACS No 8).

[...] quando os pacientes não queriam vir até a unidade, nós levávamos os potinhos na parte da manhã para eles coletar o exame em casa mesmo, ai de uma certa maneira a gente conseguiu fazer com que eles viessem, mas tem aqueles bem resistentes [...] (ACS No 4).

Nossa busca é essa, chega nessa casa, conversa com eles, trazer eles até a unidade, mas muitas vezes se perde no caminho [...] (ACS No 2).

Os dois últimos relatos relevam a importância da participação e corresponsabilização do doente para o sucesso do diagnóstico da TB. Nem sempre o SR é identificado durante a visita. Algumas vezes, o ACS é chamado pelo familiar por causa do aparecimento de sintomas, e realiza os encaminhamentos necessários.

[...] nós vamos na casa da pessoa, se ela reclamou que está tossindo a tanto tempo né? Ai a gente passa para a UBS ou pede que a pessoa venha até a UBS, se ela é acamada a gente vai levar os potinhos para fazer o escarro, pede para o paciente levar ao Dr. [médico do programa de controle da tuberculose], e lá eles vão fazer o restante dos exames (ACS No 1 ).

As ações de busca pelos ACS envolvem permanente articulação com a rede de saúde. O doente pode não querer se expor a outras pessoas e busca o maior sigilo da situação. De outra parte, pode ter problemas na locomoção para entrega das amostras no laboratório municipal. Diante dessas singularidades, o ACS faz essa mediação.

[...] já foi o caso de eu ligar direto para o Dr., mandar o paciente direto lá porque era um paciente relapso, que não se cuidava, vinha de uma história, já de vários meses com sintomas, fazendo febre a noite e suores, [...] falei direto com ele [médico] ele resolveu, está se tratando (ACS No 12). 
O trabalho do ACS caracteriza-se pela legitimidade e aceitação da comunidade, pela abordagem ampliada da família, pelo estabelecimento e manutenção do vínculo e pela articulação com os profissionais e unidades de saúde, constitui-se num elemento essencial na identificação do SR para o diagnóstico da TB.

\section{Conexão entre o território e a rede de atenção à tuberculose}

O encaminhamento é uma das principais atividades desenvolvidas pelo ACS na BSR no território, reconhecido por todos os atores de saúde; contudo, ainda a forma de organização da UBS (entrega de fichas e centralização das solicitações de exames no profissional médico), falta de alteridade na relação profissional-doente, escassez de recursos humanos (profissional médico) e fragilidade na estrutura física e na referência e contrarreferência, constituem-se em barreiras para o diagnóstico da TB na rede de ABS.

[...] a gente encaminha para consulta, pode nem ser com o médico a consulta, pode ser com a enfermagem, a enfermagem encaminha para o médico e fazem os pedidos dos primeiros exames para buscar o diagnóstico (ACS No 5).

[...]o que dificulta é conseguir ficha para ser atendido, porque as vezes eu encaminho e não tem ficha [...] (ACS No 10).

[...] acho que o pessoal [UBS] não está preparado para receber pessoas com tuberculose, porque se chega alguém aqui com sintomas, tossindo, os próprios funcionários, já ficam se cuidando (ACS No 3).

[...] nem todos [profissionais de saúde] têm essa disponibilidade de querer, de fazer visitas domiciliares, de conversa, a parte humana mesmo, aquela parte não técnica [...] de se envolver [...] infelizmente é assim [...] (ACS No 9).

Ele [doente] se sente diferente, eu assisti que o médico disse a fulano: ele é portador, pegue ele, bote lá em uma sala, deixe ele lá que depois eu vou ali, deixa ele me esperando; [...] teve um caso aqui que o cara até foi embora, [...] depois nós temos que ir buscar aquele paciente (ACS No 8).

[...] a gente não tem uma resposta se ele tem tuberculose ou não, isso não retorna para os agentes, pode até retornar para o posto, mas para a gente não retorna nada [...] se tu não for na casa dele perguntar [...] eu não sei se ele tem ou não tuberculose (ACS No 1).

O diagnóstico da TB envolve necessariamente a integração de diversos níveis de atenção, principalmente dos níveis primário e secundário. Observou-se uma permanente preocupação do ACS quando à necessidade de consolidar o elo por meio de uma efetiva comunicação entre a UBS e a Secretaria de Saúde que possibilite um efetivo acompanhamento do doente, desde a identificação SR, o acompanhamento do tratamento até a conclusão da quimioterapia.

[...] tem que trocar informações [...] a gente já comentou sobre isso, que precisava de um elo de informações, entre eles lá, a UBS e os agentes [...] (ACS No 1).

Acho que ter que ter um elo entre o posto de saúde e a secretaria de saúde, eu to falando isso há milhões de vezes aqui nas reuniões, porque se não for assim tu não vai andar [...] (ACS No 3).

Apesar das fragilidades da rede de saúde identificou-se eficiência na oferta de insumos para a realização de exames de diagnóstico. Após a entrega do material de coleta, o doente fica responsável por levar a amostra coletada até o laboratório municipal.

[...] quando é pedido o BK, já é feito, já é entregue os recipientes é só coletar e levar para o laboratório [...] (ACS No 5).

[...] facilita o acompanhamento que a gente tem da enfermagem, que geralmente vai conos$c o$, a orientação que eles dão [...] a gente tem o respaldo da secretária [...] (ACS $\left.N^{o} 4\right)$. 
O ACS releva a cumplicidade dos profissionais de enfermagem e da rede de saúde no processo de BSR e no diagnóstico da doença.

\section{O risco e a superação de medos e dificuldades}

O processo de trabalho do ACS é permeado por riscos inerentes à prática profissional. Ao reconhecê-los desenvolve suas ações com responsabilidade e comprometimento. Pelas características de seu processo de trabalho, reconhece-se como o ator com maior risco de contágio da doença, pela sua proximidade no cotidiano com as pessoas doentes na comunidade.

[...] um paciente está com tuberculose e ele não me diz, eu sei por que a outra agente me falou [...] a gente se expõe muito, porque tu não sabe [...] já aconteceu da gente tá numa casa, o paciente tá com tuberculose e a criança não fez a vacina da tuberculose, a gente até meio se apavora, e conseguimos trazer à criança para fazer a vacina [...] (ACS No 3).

[...] ir atrás mesmo, eu não deixo, eu fico em cima mesmo, tem que fazer [o exame de baciloscopia], tem que tratar [...] (ACS No 12).

Além dos riscos inerentes ao trabalho do ACS, a BSR realizada por ele envolve o enfrentamento da situação social do doente, destacando a complexidade do desenvolvimento desta ação.

[...] nós temos alguns casos de usuários de droga, é um pouco mais complicado, eles não têm esse vínculo com a unidade, eles não gostam de vir na unidade, então mesmo assim a gente consegue fazer com que eles façam o exame da tuberculose (ACS No 6).

[...] na área tem bastantes casos mesmo, porque tem muita droga, então ali é difícil da gente conseguir chegar até eles, mas eu acho que é assim, é orientado o familiar, para tentar conscientizar e fazer com que aquele que está com suspeita, vai fazer o exame (ACS No 12) (12).
A singularidade do doente pode, em alguns momentos, representar dificuldade para o ACS no desenvolvimento do processo investigativo, podendo prejudicar o diagnóstico precoce da TB.

\begin{abstract}
Eles não vem diretamente procurar [...] eles podem esconder, eles não são de se abrir [...] a gente até tem bastante informação sobre isso, só que o ruim é isso que eles não te dizem, ai tu tem que vir por fora, tratando de outras coisas para conscientizar aquela pessoa [...] tem que saber lidar com a pessoa (ACS No 11).
\end{abstract}

O ACS fez apontamentos relevantes quanto aos aspectos que precisam melhorar na rede de saúde para potencializar as ações de BSR (abordagem a família, ética, privacidade no atendimento, acesso à UBS, numero de atendimentos, reuniões internas de equipe e com o Programa de Controle da TB e cursos ou capacitações).

O que poderia melhorar realmente nessa situação é a abordagem ao paciente com tuberculose [...] quando ele chega aqui ele precisa ser abraçado pela equipe, acho que a equipe pode ajudar nessa questão, na questão da ética, da privacidade daquele paciente, acho que isso é bastante interessante, que a equipe abrasasse junto, que a gente conseguisse viabilizar mais a entrada deles na unidade [...] (ACS No 7).

[...] deve ter mais fichas, se fosse mais quantidade de ficha para eles atenderem as pessoas porque a maioria chega e não tem mais ficha e vai embora com a mesma situação que estava (ACS No 10).

Acho que o retorno dessas reuniões [reuniões de equipe com o Programa de Controle da TB] seria bem produtivo [...] acho que também mais cursos alguma coisa assim que às vezes a gente tem só um pouquinho só para substituir o necessário e uma coisa mais a fundo a gente não tem bem [...] (ACS No 4).

As falas acima revelam aspectos signifi- 
cativos no processo de BSR que incluem a superação das questões inerentes ao próprio ator de saúde e aspectos relacionados à singularidade do doente e estrutura e funcionamento da rede de saúde.

\section{DISCUSSÃO E CONCLUSÕES}

Todos os planos nacionais e consensos que se seguiram à expansão das atividades de combate à TB, enfatizaram a integração à $\mathrm{ABS}$ por meio da ESF e, em específico, do Programa de Agentes Comunitários de Saúde (PACS) das ações de controle da TB. Neste cenário a contribuição do ACS para o processo de transformação social é inquestionável (12). Percebe-se nos relatos (ACS No 3 e ACS No 4) o comprometimento dos ACS com a BSR de TB na comunidade. Embora reconheçam o risco de contrair a TB durante as visitas domiciliares, isto não se constitui em barreira para o desenvolvimento das ações de busca.

Estudo realizado no Espírito Santo identificou que a infecção pelo bacilo da TB entre os ACS foi significativamente maior do que entre seus familiares, revelando o risco ocupacional inerente à profissão (13). ACSs de São Carlos/SP investigados foram capazes de reconhecer sua participação nas ações no controle da TB; no entanto, identificaram-se fragilidades relacionadas ao conhecimento de questões de tratamento e formas de transmissão da doença, sendo necessários maiores esclarecimentos sobre a TB (9).

Destaca-se que as similaridades contextuais, onde ACS e SR estão inseridos, tornamse elementos fundamentais para o ACS se reconhecer como ator indispensável ao SR -seu semelhante. Desta forma, extrapolam seus medos e fazem suas ações acontecerem. Por serem integrantes da comunidade, tendo como local de trabalho o território onde vivem, os ACS transitam entre o saber popular e o científico, o que lhe confere singularida- de, capaz de potencializar seu trabalho. Assim como o reconhecimento da TB como um problema com raízes sociais, o que viabiliza sua atuação como agente transformador nas visitas domiciliares, fazendo a conexão entre a comunidade e a equipe da unidade de saúde $(5,14)$.

Dentre o conjunto de ações que se fazem necessárias à BSR, na comunidade, relevase no depoimento (ACS No 5) um elemento que permeia todo o processo - o vínculo do trabalhador com a pessoa doente-, estabelecido desde seu primeiro contato no domicílio. O vínculo desperta uma permanente preocupação dos ACS pelo individuo e família, buscando fornecer formas de resolutividade do problema de saúde. A atuação do ACS é capaz de fortalecer o vínculo dos usuários com a unidade de saúde e potencializar o cuidado de forma integral, direcionado para as necessidades de saúde e não apenas para a resolução da queixa. Estudo (15) identificou que os ACS sentem-se preparados para o desenvolvimento das ações individuais e coletivas de busca ativa dos SR.

Esse reconhecimento favorece a alteridade na relação do ACS com o indivíduo SR, permitindo desvendar a história do sujeito e obter informações para a identificação dos sintomas. Na posição de trabalhadores de saúde têm-se a responsabilidade social de lidar com necessidades, dores e sofrimentos do outro. Nesse processo de interação é essencial a alteridade, a compreensão dessa relação "Quem é esse outro com quem me relaciono? Como me relaciono com ele? e Para quê?" (16).

A alteridade "produzida no cotidiano do trabalho em saúde pode trazer elementos para repensar o acolhimento enquanto estratégia de mudança de modelo assistencial" (16). Nessa linha de pensamento, considerase que o debate da alteridade poderia contribuir no repensar do acolhimento ao sujeito SR que procura a UBS como resultado da efetividade das ações realizadas no território pelo ACS. As estratégias utilizadas pelo 
ACS para a aceitabilidade das suas orientações focam-se principalmente na valorização da gravidade da doença e na necessidade da busca por cuidados de saúde formais.

A qualidade do cuidado fornecida pelo trabalhador na UBS é influenciada pela sua formação e preparo para lidar com a doença e seu portador. A falta de acolhimento evidenciada nos relatos dos entrevistados (ACS No 3,8 e 9) identifica dificuldade em lidar com o doente repercutindo na oferta e recebimento das ações e serviço de saúde para diagnóstico. No processo de atenção ao SR é fundamental a prática do acolhimento pelo ACS, no sentido de receber, escutar e dar uma resposta positiva, capaz de resolver as necessidades de saúde da família (17).

Diversos fatores podem influenciar no acolhimento e continuidade das ações pelos profissionais aos SR. Dentre eles, a precarização das relações de trabalho, a incipiência de um plano de cargos e salários, e o distanciamento de uma prática integral e resolutiva, o que abre caminho para o desinteresse e o descompromisso dos trabalhadores com o sistema (18). Ademais, a ABS ainda é marcada por problemas de organização no nível operacional (dificuldade de agendamentos de consultas) (19), bem como na dimensão macro por problemas de gestão, de estruturação física, de qualificação e suficiência de profissionais gestores e trabalhadores (20).

Embora a $\mathrm{AB}$ tenha o propósito de trabalho interdisciplinar, e o ACS seja parte fundamental nesse grupo de trabalhadores, ainda há pouca interação entre os profissionais, que estão presos à prática do modelo biomédico, com a fragmentação dos saberes e do trabalho (6). Fator de entrave para potencializar as ações desenvolvidas pelo ACS no território e promover a continuidade dos cuidados.

Sem dúvidas, o ACS é ator essencial para fazer avançar a mudança direcionada para um modelo assistencial que reafirme os princípios e diretrizes do SUS, assim como o acolhimento, a formação de vínculo e a partici- pação popular nas ações em saúde (14).

Diante disso, se reconhece que a superação das dificuldades operacionais da $\mathrm{AB}$ envolvem avanços relacionados à gestão e a formação da equipe de saúde como um todo, para efetivar o trabalho interdisciplinar direcionando-o às necessidades de saúde da população. Sendo assim, há necessidade de fomentar a capacitação dos ACS, não apenas focada nos aspectos clínicos, mas principalmente na apropriação de conhecimentos da psicologia, sociologia e antropologia, visando ampliar o leque de compreensão do contexto onde a doença é produzida. Estudo (21) verificou que o treinamento e a sensibilização da equipe de SF foi capaz de promover o aumento da detecção de casos de TB, favorecendo o efetivo controle da doença.

As limitações do estudo se referem aos dados provenientes dos sujeitos estudados, já que o instrumento investigou apenas a dimensão valorativa dos entrevistados.

Considera-se que o objetivo proposto foi alcançado identificando-se que dentre as ações de busca de casos de TB no território, o ACS reconhece a necessidade do enfrentamento da situação social dos SR e valoriza a formação de vínculo para a resolução das necessidades de saúde dos SR. Quanto à articulação com o serviço de $\mathrm{AB}$ para continuidade das ações de diagnóstico da TB, identificouse fatores que limitam a sua efetivação, tais como a deficiência de estrutura e recursos humanos nas unidades de saúde.

Da apreciação do conjunto dos resultados obtidos, entende-se que os ACS investigados são atores chaves no enfrentamento da doença, capazes de construir pontes entre os serviços de saúde e a comunidade; de desenvolver uma abordagem ao indivíduo e a família baseada no vínculo, na responsabilização e resolutividade dos problemas de saúde; e produzir ações de saúde no contexto familiar, promovendo a integração do conhecimento científico e do saber popular. 


\section{REFERÊNCIAS}

1. Brasil. Ministério da Saúde. Panorama da tuberculose no Brasil: indicadores epidemiológicos e operacionais. Secretaria de Vigilância em Saúde, Departamento de Vigilância das Doenças Transmissíveis. Brasília: Ministério da Saúde; 2014.

2. Brasil. Ministério da Saúde (MS). Programa Nacional de Controle da Tuberculose. Manual de recomendações para o controle da tuberculose no Brasil. Ministério da Saúde; 2011.

3. Oliveira MF, Arcêncio RA, Ruffino-Netto A, Scatena LM, Palha PF, Villa TC. A porta de entrada para o diagnóstico da tuberculose no sistema de saúde de Ribeirão Preto/SP. Rev. Esc. Enferm. USP. 2011; 45(4): 898-904.

4. Moreira TR, Zandonade E, Maciel ELN. Risk of tuberculosis infection among community health agents. Rev Saude Publica. 2010; 4(2): 332-38.

5. Pinto ESG, Scatolin BE, Beraldo AA, Andrade RLP, Silva-Sobrinho RA, Villa TCS. O Agente comunitário de Saúde na detecção de casos de tuberculose. Cienc Cuid Saude 2014; 13(3): 519-526.

6. Klebian LVA, Acioli S. A visita domiciliar de enfermeiros e agentes comunitários de saúde na estratégia de saúde da família. Rev Eletr. Enf. 2014; 16(1): 161-9.

7. Scatolin BE, Pinto ESG, Arcêncio RA, Andrade RLP, Wysocki AD, Ponce MAZ, et al. Busca de pacientes sintomáticos respiratórios: atuação do agente comunitário de saúde no controle da tuberculose em município de grande porte, Brasil. Texto contexto-enferm. 2014; 23(2): 261-9.

8. Dantas DNA, Silva MPM, Oliveira DRC, Enders BC, Paiva REA, Arcêncio RA. Ações do agente comunitário de saúde no diagnóstico da tuberculose pulmonar. Rev Rene. 2011; 12(n.esp.): 980-7.

9. Sobrinho ECR, Freitas KG, Figueiredo RM, Caliari JS. A tuberculose na estraté- gia de saúde da família: o conhecimento dos agentes comunitários de saúde. Rev. Eletr. Enf. 2013; 15(2): 416-21.

10. Minayo MCS. O desafio do conhecimento: pesquisa qualitativa em saúde. 11a ed. São Paulo: Hucitec; 2010.

11. Bardin L. Análise de conteúdo. 4a ed. Trad. Luis Antero Reto e Augusto Pinheiro. Lisboa: Edições 70; 2004.

12. Pinto AAM, Fracolli LA. O trabalho do agente comunitário de saúde na perspectiva da promoção da saúde: considerações práxicas. Rev. Eletr. Enf. 2010; 12(4): 766-9.

13. Rodrigues PM, Moreira TR, Mores AKL, Vieira RCA, Dietze R, Lima RCD, et al. Mycobacterium tuberculosis infection among community health workers involved in TB control. J Bras Pneumol. 2009; 35(4): 351-8.

14. Morosini MV. Educação e trabalho em disputa no SUS: a política de formação dos agentes comunitários de saúde. Rio de Janeiro: EPSJV; 2010. 202 p.

15. Guerrero P, Mello ALSF, Andrade SR, Erdmann AL. O acolhimento como boa prática na atenção básica à saúde. Texto contexto-enferm. 2013; 22(1): 132-40.

16. Matumoto S, Mishima SM, Fortuna CM. Acolhimento: uma reflexão acerca da alteridade na relação entre trabalhador e usuários no trabalho da rede básica de saúde. En: Proceedings 8. Simp. Bras. Comun. Enferm [Internet]; 2002 May 02-03; São Paulo, SP, Brazil. 2002 [citado 10 may 2013]. Disponível: http://www.proceedings.scielo. br/scielo.php?script $=$ sci_arttext $\&$ pi d=MSC0000000052002000100044\&ln$\mathrm{g}=\mathrm{en} \& \mathrm{nrm}=\mathrm{van}$.

17. De Carli R, Costa MC, Silva EB, Resta DG, Colome ICS. Acolhimento e vínculo nas concepções e práticas dos agentes comunitários de saúde. Texto contexto-enferm. 2014; 23(3): 626-32.

18. Assis MMA, Alves JS, Santos AM. Políticas de recursos humanos e a (re) signifi- 
cação das práticas dos trabalhadores no Sistema Único de Saúde (SUS): apontamentos para o debate. Rev Baiana de Saúde Pública 2008; 32: 7-14.

19. Clementino FS, Miranda FAN. Acessibilidade: identificando barreiras na descentralização do controle da tuberculose nas unidades de saúde da família. Rev. Enferm. UERJ. 2010; 18(4): 584-90.

20. Silveira DS, Facchini LA, Siqueira FV, Piccini RX, Tomasi E, Thumé E, et al. Gestão do trabalho, da educação, da informação e comunicação na atenção básica à saúde de municípios das regiões Sul e Nordeste do Brasil. Cad Saude Publica. 2010; 26(9): 1714-26.

21. Façanha MC, Melo MA, Vasconcelos FF, Sousa JRP, Pinheiro AS, Porto IA, Parente JM. Health team training and active community surveillance: strategies for detection of TB cases. J Bras Peneumol. 2009; 35(5): 449-454. 